\title{
Alternative management of proximal aortic dissection: concept and application
}

\author{
Xun Yuan $^{1,2} \cdot$ Andreas Mitsis $^{3} \cdot$ David Mozalbat $^{4} \cdot$ Christoph A. Nienaber ${ }^{1,2}$
}

Received: 3 August 2021 / Revised: 20 September 2021 / Accepted: 21 September 2021 / Published online: 13 December 2021

(c) The Author(s) 2021

\begin{abstract}
Open surgery remains the mainstay of treatment for acute type A aortic dissection and should be offered to most patients. However, there are elderly patients in which surgical treatment may be deemed extremely high risk or futile. Endovascular treatment approaches have been applied to a small number of these patients and data are limited to case reports and small series. The application of endovascular therapies to ascending aorta is currently limited by anatomical and technical challenges posed by the dynamic motion of the ascending aorta and the proximity of vital structures to intended landing zones (aortic valve, coronary arteries, and supra-aortic branches) and lack of specially designed endografts to address these issues. While thoracic endovascular aortic repair (TEVAR) has replaced open aortic repair for a suitable lesion in distal aortic dissection, some selected patients with type A aortic dissection at high surgical may be candidates. Hence, there is potential because, in proximal (Stanford type A) dissections, 10-30\% of patients are not accepted for surgery, and 30-50\% are technically amenable for TEVAR. Recent experience has shown that carefully selected patients with favorable anatomical characteristics may be subject to endovascular stent-graft treatment as a last resort with mixed results. Technical improvement is necessary to offer. satisfactory endovascular options in non-surgical candidates.
\end{abstract}

Keywords Proximal aortic dissection · TEVAR $\cdot$ Endovascular $\cdot$ Occluder $\cdot$ Coils

\section{Introduction}

Acute type A aortic dissection (TAAD) is a surgical emergency with high morbidity and mortality. With early mortality varying from 17 to $26 \%$, TAAD continues to represent a great challenge for aortic surgeons [1,2]. Without repair, mortality can reach up to $50 \%$ within the first $48 \mathrm{~h}$ [3]. The primary aim of surgery is the prevention of death from aortic rupture and is mainly accomplished by excision of the proximal tear, supracoronary aortic replacement, and

Christoph A. Nienaber

c.nienaber@rbht.nhs.uk

1 Cardiology and Aortic Centre, Royal Brompton and Harefield Hospitals, Guy's and St Thomas' NHS

Foundation Trust, London SW3 6NP, UK

2 National Heart and Lung Institute, Faculty of Medicine, Imperial College London, London, UK

3 Cardiology Department, Nicosia General Hospital, Strovolos, Cyprus

4 Cardio-Thoracic Surgery Department, St George Hospital London, London, UK re-establishment of dominant flow to the distal true lumen. Although improvements in cardiopulmonary bypass, cardiac surgery techniques, and cerebral protection have been associated with better outcomes, perioperative mortality ranges from 15 to $30 \%$ particularly in the elderly with significant comorbidities [4]. In addition, neurological complications $(18 \%)$ remain high. While patients' outcome is largely determined by comorbidities and dissection-related complications, surgical mortality is high in unstable patients with pre-operative organ malperfusion.

Almost 10-30\% of the patients are not accepted for surgery because they are considered unfit to undergo open repair [3] Furthermore, coma, shock secondary to pericardial tamponade, malperfusion of coronary or peripheral arteries, and stroke are important predictive factors for post-operative mortality. Recent image analysis had suggested that up to $50 \%$ of patients with proximal dissection may be technically amenable to thoracic endovascular aortic repair (TEVAR) $[5,6]$. With the ultimate goal of fully catheter-based interventions to the ascending aorta, we have screened the current literature for this review. 


\section{Favorable anatomical characteristics and technique}

Achieving safe and successful endovascular access for the deployment of stent-graft devices is the most important step during TEVAR. Femoral artery access is the standard for TEVAR and is feasible in at least $70 \%$ of cases. However, due to severe tortuosity, both transapical and trans-carotid access may be explored.

An entry tear located in the center portion of the ascending aorta offers the best target for TEVAR, as the absence of an acceptable proximal landing zone is the most common exclusion criterion. Entry tears close to coronaries or aortic valves are prohibitive as is aortic regurgitation. A length of a proximal or distal aortic landing zone more than $20 \mathrm{~mm}$ is required in order to deploy safely. Entry tears close to the brachiocephalic artery would require complex branching/ fenestration strategies; significant aortic valve regurgitation is prohibitive today, but future technology may combine a stent-graft with an integrated valve. Currently, only a limited number of choices are available regarding the type of stentgraft and delivery system as relatively large diameter and short length stent-grafts are required.

\section{Complications}

Both, anatomic complexity and motion of ascending aorta are major obstacles to the use of endovascular technologies. Limitations arise from the short and wide shape of the ascending aorta that often curves sharply, resulting in marked disparity in the lengths of its inner and outer curves.

Complications of proximal TEVAR are neurologic as a result of brain ischemia or embolization. Excessive wire and catheter manipulation in a diseased aortic arch with risks of air embolism from deployment systems and inadvertent coverage of arch branches increase the risk of stroke. In one report, stroke occurred in three of 45 patients $(6.8 \%)$ - two of which had received their stents via left common carotid artery approach [7,8].

Another important characteristic relates to the design of stent-graft; devices without proximal bare springs are recommended to avoid compromising of the aortic valve.

Supra-aortic vessel occlusion is another important concern. If the distance between the distal landing zone of the stent-graft and the origin of the supra-aortic vessels is not adequate, the procedure can only proceed with arch vessel rerouting or bypass to generate sufficient anchoring area. Alternatively, debranching of the innominate trunk to the left common carotid artery has been used to increase the length of the distal landing zone.

Proximal type I endoleak is a common complication after stent-grafting. Rapid ventricular pacing is probably required to avoid misplacement and windsock effect and assure precise stent-graft placement [9]. The diameter of the stent-graft should be according to the previous aortic dimension (before dissection) to avoid oversizing. With the goal to re-shape the dissected ascending aorta, cover the entry tear and depressurize the false lumen (FL). It is accepted that a totally thrombosed FL is linked to better long-term survival after TEVAR [10]. In the presence of endoleaks, balloon expansion for better wall apposition or even a short extending stent or occluder can be used to seal the endoleak. Sometimes additional procedures including coils or other devices are necessary.

\section{Our experience}

Between the year 2015 and 2020, 19 patients with acute, subacute, or chronic TAAD with the proximal entry tear located between the coronaries and brachiocephalic artery were treated with TEVAR. Various stent-graft configurations were used to seal the proximal entry tear in the ascending aorta under rapid pacing. Procedural success was achieved in $17 / 19$ patients $(89.5 \%)$. There was one intra-procedural death and one minor stroke, but no additional deaths at 30 days. At 36 months, there were 4 further deaths (all from non-aortic causes). The mean survival of these 4 deceased was 23 months (range 15-36 months) [11]. Follow-up computed tomography (CT) demonstrated favorable aortic remodeling except for one case where a new proximal type I endoleak was discovered after 18 months and the patient was treated with high-risk open surgery (Fig. 1).

Procedural planning was based on contrast-enhanced, electrocardiograph-gated CT, which was evaluated with standard image processing software to select the appropriate stent-graft size. The stent-grafts used were usually ZENITH TX2 (Cook, Bloomington, Ind), GORE C-Tag (Gore Ltd, London, UK), or Relay NBS (Bolton, Barcelona, Spain). They are made of a self-expanding Nitinol stent platform covered with polyester fabric mounted onto a catheter-based delivery system.

With the patient under general anesthesia, a temporary pacing wire was placed in the right ventricle (RV) and vascular access for the TEVAR device (22-24 F) was obtained via femoral arterial cut-down. The true lumen of the aorto-iliofemoral arterial route was navigated using a soft long hydrophilic guidewire (Terumo Medical Corp, Somerset, NJ) protruding ahead of a pigtail catheter to reach the left ventricle under fluoroscopy [12], then exchanged for a stiff wire. The stent-graft was then delivered over the stiff guidewire to its intended position, where its distal landing zone is between distal to coronary ostia and proximal to brachiocephalic artery in the ascending aorta. In this position, the distal tip of the delivery system may cross the aortic valve. For stentgraft deployment, rapid right ventricular pacing at $180 \mathrm{bpm}$ 
Fig. 1 A example of late complication of thoracic endovascular aortic repair (TEVAR) in proximal aortic dissection. A shows a localized type A aortic dissection; B shows the excellent result after TEVAR with a short stent-graft; $\mathbf{C}$ shows a stent-induced re-entry tear (erosion) from the proximal contact between the crown of stent-graft and out-curve of the aorta after 6 months

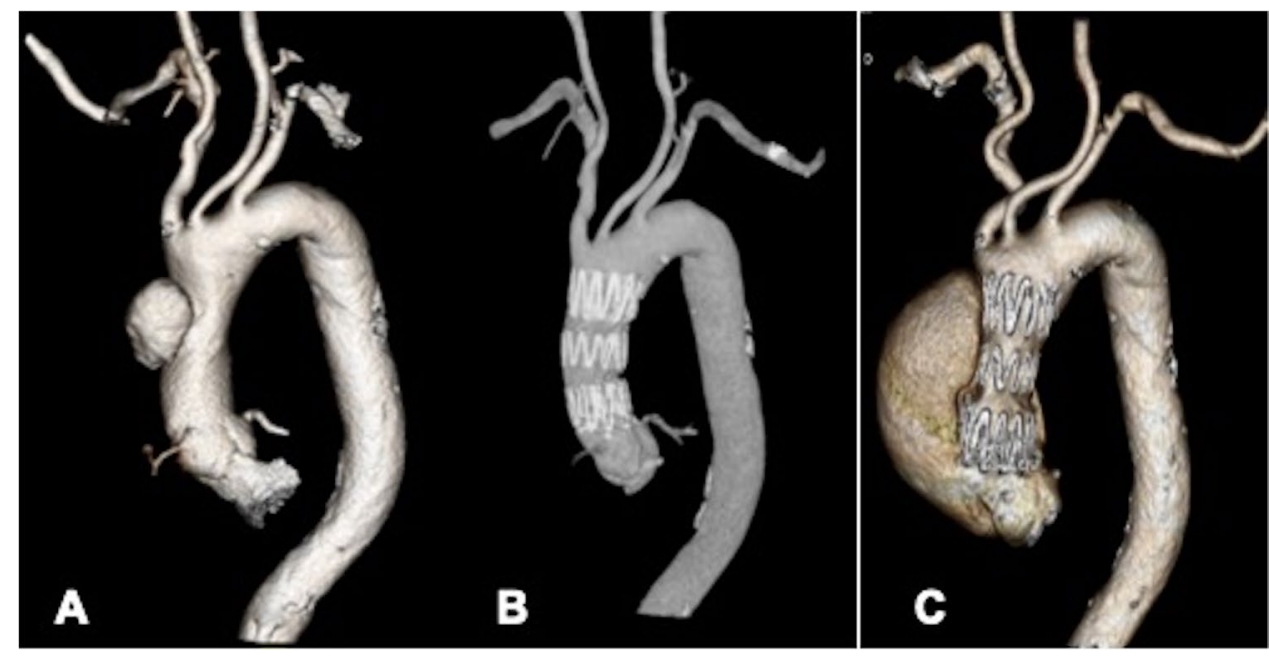

was used to reduce systolic blood pressure to $50 \mathrm{~mm} \mathrm{Hg}$ to avoid displacement (by windsock effect) during deployment. At the end of the procedure, the temporary pacing wire was removed, the femoral artery access site closed, and the patient extubated and transferred to the coronary care unit. Procedural success was defined as successful placement of the stent-graft in its intended position with the sealing of the entry tear.

\section{FL intervention to promote remodeling and thrombosis concept}

The FL intervention to promote remodeling and thrombosis (FLIRT) concept relies on minimalistic, percutaneous interventions to manage the FL by inducing thrombosis, both proximally and distally, by the use of occluder devices, vascular plugs, distal stent-grafts, or coils and glue both in chronic type A and B dissection. The decision to proceed with percutaneous means to induce FL thrombosis and eventually remodeling was based on the presence of both (re)entries and FL flow/expansion.12 All embolization and closure procedures were performed percutaneously, under local or general anesthesia, via femoral and/or brachial access. Various percutaneous techniques were utilized for FLIRT:

1. Promotion of FL thrombosis by sealing the entry tear or communication using a combination of coils and Amplatzer ${ }^{\mathrm{TM}}$ patent foramen ovale (PFO) or atrial septal defect (ASD) occluders (as short stent-graft are not suitable).

2. Retrograde catheterization of the FL via distal re-entry tears and induction of thrombosis by coils, duct occluder in addition to true lumen scaffolding with bare metal stent and/or re-entry occlusion by short stent-graft.

3. Selective application of Onyx ${ }^{\circledR}$ glue in FL and distal sealing of re-entry site by the use of a local stent-graft.
In essence, the approach to managing proximal and distal dissection was fundamentally the same. Initially, a 5-F coronary diagnostic catheter was advanced into the target entry. If appropriate selective coiling was performed with spiral coils directly detached via 5F catheter (William Cook Europe, Bjæverskov, Denmark). In all type A cases, coiling was followed by definitive sealing of the proximal tear using a PFO or ASD-occluder (St. Jude Medical, Minneapolis, MN), delivered via a dedicated sheath or 8-F coronary guiding catheter (Fig. 2). Ethylene-vinyl alcohol copolymer (Onyx ${ }^{\circledR}$ glue, ev3, CA) was injected via conventional 6-F MP coronary guide catheter in 2 cases and performed in a state-of-the-art hybrid theatre offering the latest imaging technology and simultaneous transesophageal echocardiogram (TOE).

\section{Other reported experience}

Although currently there are no multicenter clinical trials focusing on analyzing the outcome of endovascular treatment in proximal aortic disease, there are a couple of small-scale studies that reported and summarized their own experiences in this challenging and controversial technique (Fig. 3). We analyzed 20 recently published articles with available patients' outcome dates. The number of patients enrolled in the studies ranges from 4 to 45 with a TEVAR primary success rate varies from 59.1 to $100 \%$. The mortality rate at 1 -year follow-up was noted 0 to $28.3 \%$, while the endoleak occurred and re-intervention required after TEVAR are $0-27.3 \%$ and $0-33.3 \%$, retrospectively (Table 1 ).

\section{Discussion}

Furthermore, the overall technical success of all studies on TEVAR in the ascending aorta was $95 \%$ and the pooled stroke rate was $7.8 \%$. Device and delivery system limitations 


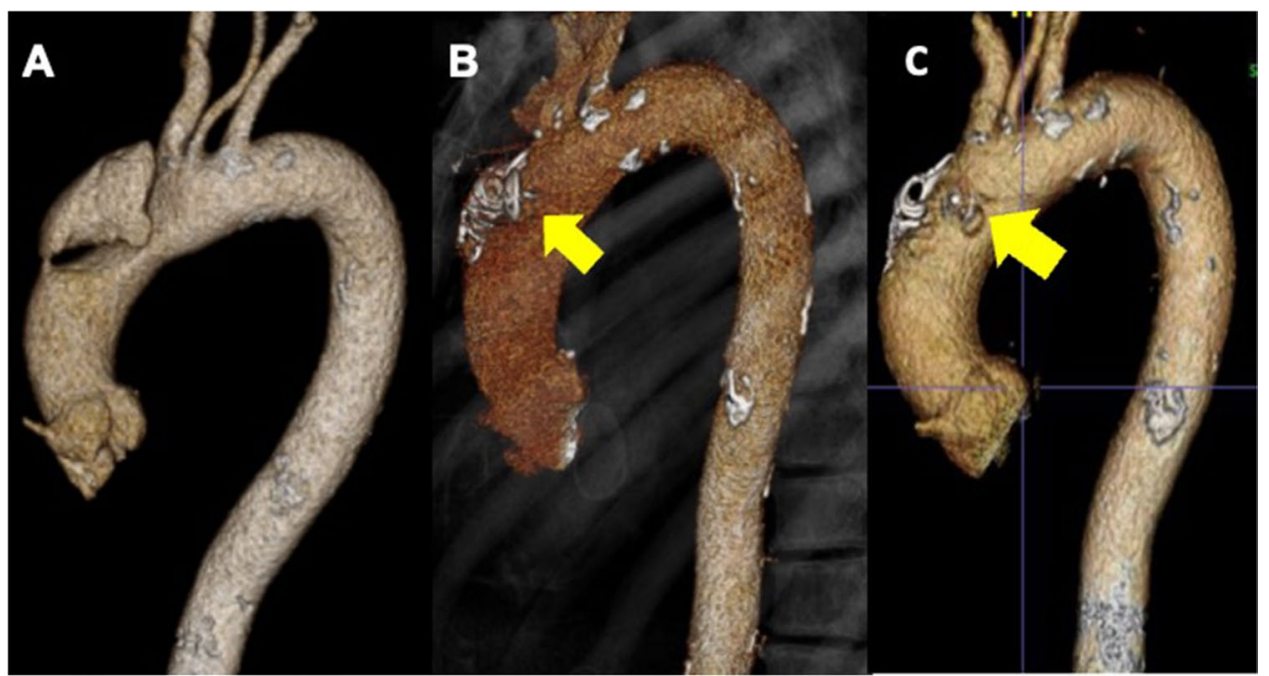

Fig. 2 An example for false lumen intervention to promote remodeling and thrombosis (FLIRT) procedure. A shows a localized type A aortic dissection with an entry tear at the outer curve of the aorta just proximal to the innominate artery. $\mathbf{B}$ shows the result of endovascular treatment with coils dropped in the false lumen to promote thrombo- sis and a patent foramen ovale (PFO) occluder (arrow) to isolate the communication between true and false lumen (FLIRT concept). C shows a complete remodeling of the aorta without any complications after 2 years of procedure

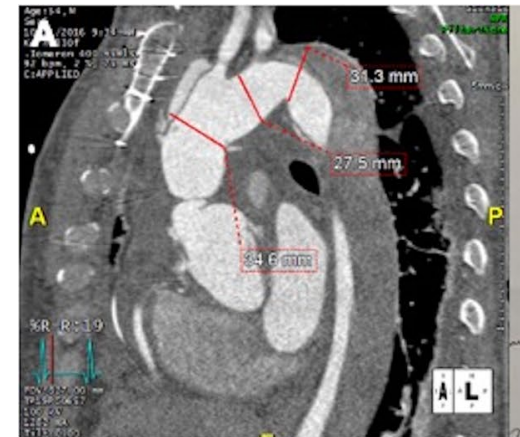

B

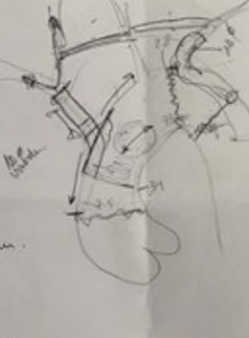

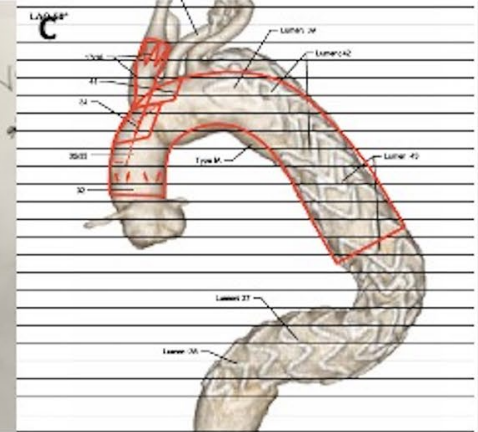

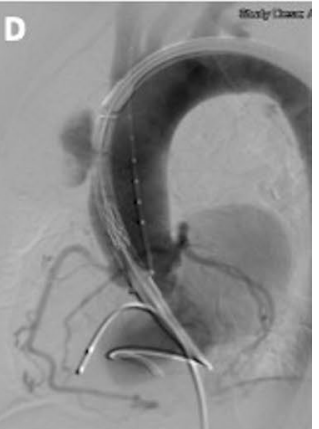

Fig. 3 A careful assessment and planning before the procedure is the key element for a successful endovascular treatment for proximal dissection. A shows an extensive measurement based on gated computed tomography (CT) images; B shows the exploration of potential pro- cedure in a sketch; $\mathbf{C}$ shows computational modeling to mimic the devices and outcome; $\mathbf{D}$ shows a real-world case accomplished after intensive multidisciplinary preparation

conclude that this fact contributed to their higher morbidity and mortality. However, complications related to coronary exclusion were responsible for only one death in this study. Additionally, the Physician-Sponsored Investigational Device Exemption (PS-IDE) protocol entails a 1-cm minimum landing zone, which was thought to be adequate for TAAD [15]. Surgeon experience and institution volume undoubtedly have a significant influence on outcomes of this technically complex approach; Table 2 summarizes the currently debated pros and cons of an endovascular approach to the ascending aorta.

Surgical mortality is reported between 10 and $25 \%$ [1, $16]$ today depending on the complexity of the operation 
Table 1 Procedural mortality—meta-analysis of TEVAR in proximal aortic dissection

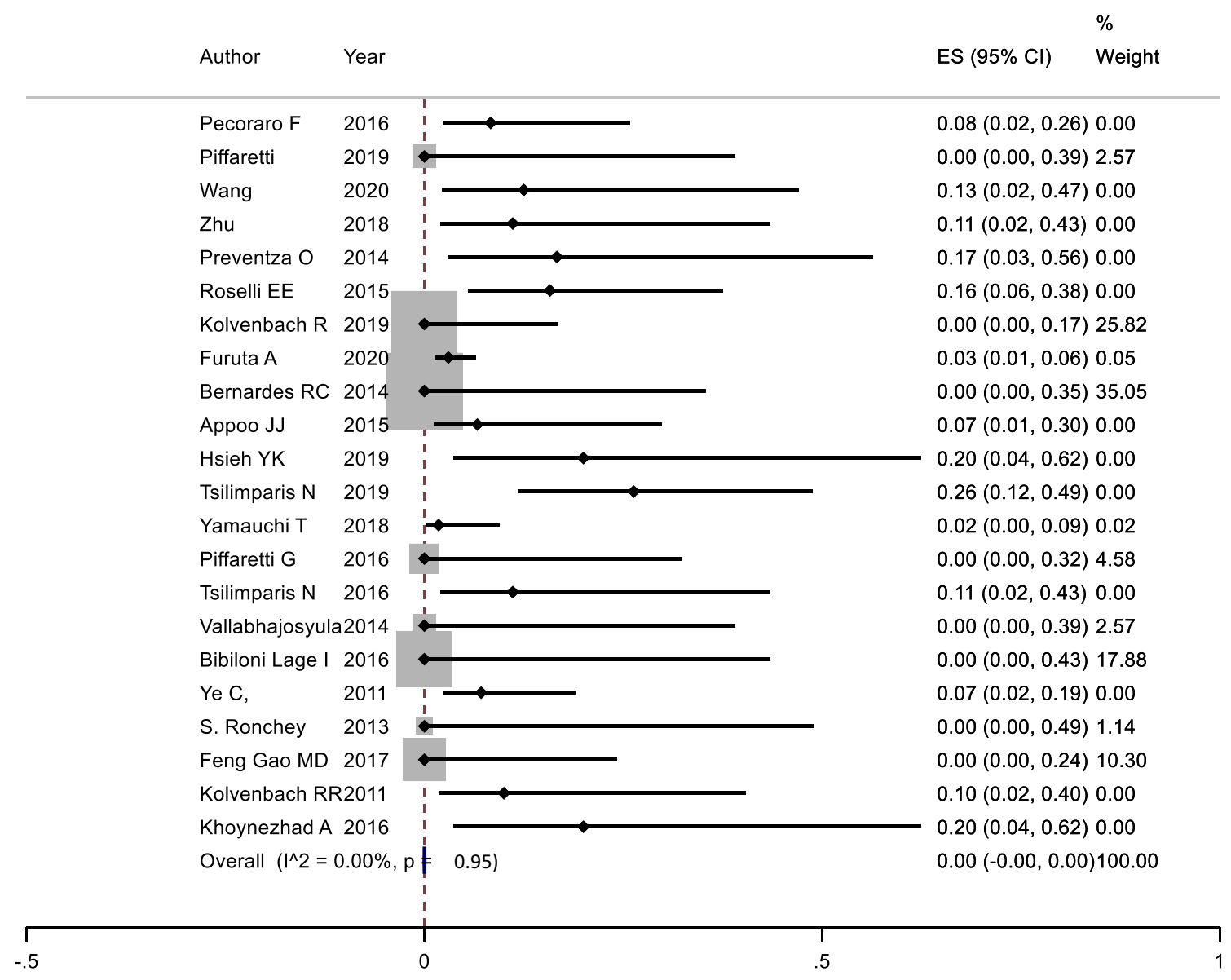

Table 2 Endovascular management of the ascending aorta

\begin{tabular}{ll}
\hline Pros & Cons \\
\hline Option for patients with extreme risk for open surgery & Highly selected patients with suitable anatomy \\
Avoidance of thoracotomy & - Entry tear central between coronaries and brachiocephalic trunk \\
Trans-arterial (femoral) or trans-apical access is feasible & - Proximal and distal sealing zones $20 \mathrm{~mm}$ \\
Applicable to select dissection, focal, and suture line aneurysm & - Relevant aortic valve regurgitation unsuitable \\
& - Shaggy aortic arch/high stroke risk \\
& - Rapid RV pacing is compulsory \\
& Device embolization of migration \\
& May require additional neck vessel rerouting \\
& Limited size and lengths of available stent-grafts \\
& Risk of damage to the aortic valve and left ventricle by wire of the delivery \\
& device \\
& Risk of erosion from the extensive 3-dimensional motion of the ascending aorta
\end{tabular}

and the clinical status of the patient. In experienced hands, the procedural mortality of TEVAR to the ascending aorta was $8 \%$, comparing favorably with published endovascular mortality of $11 \%$ [17, 18]. Interestingly, most anticipated complications such as major stroke did not occur; 1 minor stroke (transient) and 1 death from guidewire perforation of the left ventricle had led to fatal tamponade in our series. The number of TEVAR procedures performed relative to all emergency surgeries for TAAD in our 3 centers is almost $2 \%$, or $6-17 \%$ of inoperable type A cases. 


\section{Goals of an endovascular strategy}

Successful sealing of the proximal entry with no development of proximal type I endoleak is the goal and was achieved in all cases compared to $10 \%$ failure rate reported elsewhere [19] in the attempt to reshape the dissected ascending aorta and depressurize the FL $[7,20]$. Once precisely deployed, the process of remodeling of the FL appears similar in both proximal and distal dissections [21, 22]. Most of our cases were DeBakey type II dissections, and even the 2 cases of type I dissection [11] proved that the therapeutic concept of closing the entry holds true in ascending aortic dissection [23, 24]. Current experience from small case series underlines the feasibility of proximal endovascular procedures over more than 10 years [6, 20, 22, 25-27]. Our series over 6 years with a mean follow-up of $>20$ months is encouraging considering inoperability of those patients. In other words, it is feasible to avoid high-risk/complex surgery and apply less-traumatic interventions to obtain acceptable outcomes. The advantage of TEVAR includes the avoidance of thoracotomy, cardiopulmonary bypass, selective head perfusion, and associated surgical risks in a high-risk elderly population [28]. If the high initial $60 \%$ mortality of TAAD not accepted for surgery can be successfully lowered by TEVAR, such a less traumatic strategy may potentially become an option in a broader spectrum of patients [16].

TEVAR will not be feasible in every patient due to anatomic constraints. Currently, only limited choices are available regarding the type of stent-graft and delivery system as large diameter and short length stent-grafts are required. Existing delivery systems need to be modified with a short nosecone not to damage the aortic valve or cause left ventricular perforation. In the context of stent-graft delivery, rapid $\mathrm{RV}$ pacing is probably the most efficient method to avoid the windsock effect, enabling precise stent-graft placement. Transesophageal echocardiography is also useful in guiding stent-graft positioning and assessing the sealing of the entry tear [12]. It should be emphasized that a multidisciplinary team, providing surgical and interventional competence, should perform such a procedure similar to transcatheter aortic valve replacement. TEVAR in the ascending aorta can be a definitive solution for patients not accepted for surgery, or a bridging solution in case of unclear neurological scenarios (e.g., major stroke) to buy time for reconstructive surgery. It is conceivable that a combined transcatheter aortic valve and ascending graft technology may become the next technological improvement to tackle the ascending aorta.

\section{Ancillary procedures}

In patients with organ or limb malperfusion, coverage of the proximal entry tear will usually expand the true lumen and re-perfuse ischemic organs. Patency of the FL both in ascending and descending aorta is not uncommon and is considered to herald unfavorable long-term outcomes [29, 30]. In the absence of thrombosis and remodeling, further dilatation and rupture are likely [31-33]. Thus, it appears logical to induce complete FL thrombosis to fully manage aortic dissection and improve outcomes [34] in the longterm, knowing that incomplete or partial FL thrombosis and a patent FL promote further expansion and possibly subsequent rupture (RR 2.69, $P=0.002$ ) [29]. The classic endovascular approach is the occlusion of connections between true and FL by additional stent-graft coverage, or by custom-made fenestrated and branched endografts at the level of abdominal re-entries [35]. This method affords FL thrombosis throughout the dissected thoraco-abdominal aorta [36], but carries significant risks, especially for spinal cord ischemia, as many segmental arteries may be covered. Recently, alternatives were introduced including "candy-plug" and "knickerbocker" techniques, relying on FL obstruction by prohibiting distal FL backflow [37, 38]. Both techniques have drawbacks, have not been tested in larger series, and are considered more traumatic and potentially risky. Similarly, the use of multilayer stent technology would not be safe or appropriate as side branches and communications are likely to stay open $[39,40]$. More interestingly, a recent "true lumen intervention" suggests tackling distal re-entry sites by small stent-grafts to promote thrombosis of the FL [41].

Conversely, the FLIRT concept uses technology applied to the FL in order to promote remodeling; it ranges from injection of embolizing material to deployment of coils, plugs alone, or in combination, as integral components of FL intervention $[42,43]$. Onyx ${ }^{\circledR}$ is the most common embolizing agent and has previously been used with coils to promote FL thrombosis and seal endoleaks [44, 45].

In the ascending aorta, the FLIRT concept provides additional endovascular options. FLIRT has proven safe and feasible in 5 attempted cases of chronic type A dissection and is certainly less traumatic than other techniques including stenting of ascending aorta [43]. Closure of entry tears, restoration of single-lumen blood flow, and enhanced FL thrombosis may reap long-term benefits [11], without the risks of stent-grafting the ascending aorta (Fig. 2). Hence, the FL channel is an alternative pathway to allow a catheter to reach the tear to deploy coils and Amplatzer ${ }^{\mathrm{TM}}$ vascular occluder devices in the FL. In our series 2 Amplatzer ${ }^{\mathrm{TM}}$ Septal Occluders, 2 Amplatzer ${ }^{\mathrm{TM}}$ PFO Occluders, 1 Amplatzer ${ }^{\mathrm{TM}}$ Vascular Plug II, and 1 Amplatzer ${ }^{\mathrm{TM}}$ Duct Occluder II were used. The criteria to choose these devices were individualized to the anatomical characteristics of any given patient and to the access sites needed for different sizes of delivery systems of those devices. The sustainability of FLIRT as a concept to promote remodeling may be controversial and longer follow-up is certainly needed. 
Advanced interventional skills, with formal training in interventional cardiology, are also essential for safety interventions. Finally, the best timing of FLIRT as a complimentary intervention is not clear yet, but a better understanding of CT imaging may help to identify patients likely to benefit from FLIRT $[46,47]$.

\section{Technical aspects of endo-grafting the ascending aorta}

Although the intimate proximity of the aortic valve and coronary ostia necessitates precise deployment of the graft specifically in the region of ascending pathology (despite the greater forward flow forces that hinder precision), smaller landing zones do not seem to explain morbidity and mortality. Still, given the complexity of the ascending aorta and the uniqueness of the local anatomy and hemodynamic forces, the ultimate quandary is one of operability based on the currently available technology. The ability of gated CT imaging to quantify intimal defects provides crucial information for deciding which patients are candidates for endovascular approaches and for procedural planning in this delicate region. The curvature in this area of the aorta is steep and the diameter and length fluctuate significantly, explaining the difficulty in the coaxial deployment of the graft from a transfemoral approach. An alternative is the transapical approach [48, 49], which is occasionally used for cardiovascular operations such as ventricular assist devices and transcatheter aortic valve replacement. The short distance to the ascending aorta can increase the accuracy of deployment in the context of a limited landing zone, but the access is quite traumatic. Immediate pericardial drainage is possible in patients developing pericardial effusion. With further technical development and investigation of various approaches, this strategy may become important. Other options include axillary and carotid cannulation as well as transseptal access from the femoral vein. Low-profile delivery systems may broaden the feasibility and success of new approaches.

From a purely technical standpoint, dissection-specific devices are necessary for better procedural outcomes. In 2012, Metcalfe et al. [20] reported the first use of a device designed explicitly for the ascending aorta in the UK (a 34-mm-diameter Zenith Ascending Dissection device; Cook Medical, Bjaeverskov, Denmark). In the USA, the first stentgraft for use in the ascending aorta is the modified COOK TX2 Thoracic Stent-graft without proximal bare metal (with a diameter up to $46 \mathrm{~mm}$ and a treatment length up to $90 \mathrm{~mm}$ ), and the investigational short GORE Active Control device is used in the ongoing Ascend trial. Fenestrated, branched, bifurcate, and custom-made endografts may also play a role in the future of endovascular treatment of TAAD $[50,51]$. Additionally, strategies for graft delivery must be upgraded alongside the design of arch-specific stent-grafts.
Because of tortuosity encountered in traversing the iliac vessels and the arch, trackability, pushability, and torquability may be lost. Pre-curved delivery systems and specific guidewire configurations may improve coaxial deployment via the typical TEVAR transfemoral approach.

\section{Current technological limitations}

With no devices currently approved for use in the ascending aorta, there is a significant limitation of available stentgrafts. To date, all cases have received off-label devices and have been limited to select cases in which the patient's anatomy could be aligned with available sizing options. These sizing limitations illustrate the need for approved devices designed specifically to conform to the anatomic challenges of the ascending aorta.

Whereas femoral artery access is preferred, some delivery devices are not long enough to reach the ascending aorta; the axillary and carotid arteries are less likely to be affected by peripheral vascular disease and therefore can often provide viable access in patients with severely diseased femoral or iliac vessels [52]. Review of the literature showed no difference in outcomes with the use of a femoral vs. alternative access approach that can be safely performed in the hands of a skilled endovascular specialist sometimes with the use of a conduit as an access. Despite a cohort of exclusively high-risk patients, early outcomes appear promising in those individuals treated endovascularly for ascending aortic conditions. Many of the patients treated with ascending aortic stent-grafts were considered unfit for open surgery on the basis of their medical comorbidities. Aorta-related mortality was $5 \%$ and all-cause mortality was $14.9 \%$, which is significantly lower than that observed for patients with type $A$ dissections treated with open surgery, which ranges from 17 to $26 \%$ [53]. One population of patients that is poised to benefit from endovascular therapies in the ascending aorta is patients with acute TAADs. The International Registry of Acute Aortic Dissection reports that patients undergoing open surgical repair for acute type A dissections have an inhospital mortality of $26.9 \%$; moreover, up to $30 \%$ of patients are considered unfit candidates for open surgery on the basis of advanced age, medical comorbidities, and preference of the patient [54]. Endovascular approaches are an appealing prospect for these patients because they are minimally invasive, circumvent the need for cardiopulmonary bypass, and involve a much less extensive surgery. Whereas it remains to be seen whether these interventions will provide a long-term solution for these patients, one application of the technology is the stabilization of acutely sick patients who can later go on to have a definitive open repair.

The complication most commonly encountered in patients with ascending aortic stent-grafts is endoleak, with an overall rate of $18.2 \%$. The overall endoleak rate encountered in 
TEVAR ranges from 3.6 to $8.7 \%$ and represents a significant limitation of this technology [55]. Endoleak can contribute to FL expansion in dissections and increase the risk for aortic rupture. In response, a number of promising technologies including endoanchors, branched stent-grafts, and in situ fenestration are currently being used both to prevent and to treat endoleak in the abdominal aorta and aortic arch [56-58]. As adjunctive technologies continue to develop, they may in the future help overcome issues of endoleak due to poor stent-graft deployment or inadequate landing zones in the ascending aorta.

\section{Limited evidence for TEVAR}

With the majority of manuscripts consisting of case reports and small series, there remains a strong selection bias in the literature with regard to endovascular management of the ascending aorta; Table 1 is a summary of previously published evidence. Within the past 2 years, some larger studies have been published that help providing greater insight into emerging ascending aortic interventions. $\mathrm{Li}$ et al. presented one of the largest cohorts to date, with a total of 15 patients with TAAD treated endovascularly by stenting of the ascending aorta. In this cohort, long-term follow-up revealed 8 complications, including new dissection, cardiovascular ischemia, retrograde TAAD and ventricular pseudoaneurysm, pericardial effusion, kidney malperfusion, and perigraft endoleak. No deaths were reported in the follow-up period. A total of four reinterventions were documented, including branched stent-graft deployment for new dissection, coronary stenting, and one open surgical repair. Although the overall complication rate was high in these patients, close monitoring allowed early detection and successful treatment avoiding long-term mortality. In addition, these patients were originally selected to receive endovascular interventions after being deemed ineligible for open surgical repair [59] suggesting that endovascular interventions can serve as a valuable intervention for patients who are considered unfit candidates for open surgery. Nevertheless, close follow-up is needed to prevent and treat complications as these arise. Thus far, most published results are positive, but the data are based almost exclusively on single-center experiences in case reports or case series. To truly understand the outcomes associated with endovascular interventions in the ascending aorta, a prospective, randomized controlled trial should be advised and completed, ideally across multiple, high-volume aortic centers and the use of stent-graft designed specifically for the ascending aorta. With the current device limitations, this technology should be reserved for high-risk surgical candidates, particularly those denied open interventions. Interventions should be performed in select patients whose focal ascending aortic lesions can be addressed with currently available devices. In addition, these procedures should be performed by experienced endovascular specialists, and steps should be in place for open interventions when complications arise (Fig. 3).

\section{Summary and outlook}

TEVAR in proximal aortic dissection can be performed with high technical success and acceptable morbidity and mortality in high-risk inoperable patients. Optimal aortic remodeling could be achieved by using new short-length stent-graft devices or ancillary technology like FLIRT. The current iteration of stent-graft technology however needs to be adapted to the specific challenges of the ascending aorta.

\section{Declarations}

\section{Ethics approval NA}

Informed consent Written consents have been obtained from patients whose medical images were used in this article.

Conflict of interest The authors declare no competing interests.

Open Access This article is licensed under a Creative Commons Attribution 4.0 International License, which permits use, sharing, adaptation, distribution and reproduction in any medium or format, as long as you give appropriate credit to the original author(s) and the source, provide a link to the Creative Commons licence, and indicate if changes were made. The images or other third party material in this article are included in the article's Creative Commons licence, unless indicated otherwise in a credit line to the material. If material is not included in the article's Creative Commons licence and your intended use is not permitted by statutory regulation or exceeds the permitted use, you will need to obtain permission directly from the copyright holder. To view a copy of this licence, visit http://creativecommons.org/licenses/by/4.0/.

\section{References}

1. Trimarchi S, Nienaber CA, Rampoldi V, et al. Contemporary results of surgery in acute type A aortic dissection: The International Registry of Acute Aortic Dissection experience. J Thorac Cardiovasc Surg. 2005;129:112-22.

2. Tsai TT, Trimarchi S, Nienaber CA. Acute aortic dissection: perspectives from the International Registry of Acute Aortic Dissection (IRAD). Eur J Vasc Endovasc Surg. 2009;37:149-59.

3. Pape LA, Awais M, Woznicki EM, et al. Presentation, diagnosis, and outcomes of acute aortic dissection: 17-year trends from the International Registry of Acute Aortic Dissection. J Am Coll Cardiol. 2015;66:350-8.

4. Trimarchi S, Eagle KA, Nienaber CA, et al. Role of age in acute type A aortic dissection outcome: report from the International Registry of Acute Aortic Dissection (IRAD). J Thorac Cardiovasc Surg. 2010;140:784-9.

5. Sobocinski J, O’Brien N, Maurel B, et al. Endovascular approaches to acute aortic type A dissection: a CT-based feasibility study. Eur J Vasc Endovasc Surg. 2011;42:442-7. 
6. Ihnken K, Sze D, Dake MD, Fleischmann D, Van der Starre P, Robbins R. Successful treatment of a Stanford type A dissection by percutaneous placement of a covered stent graft in the ascending aorta. J Thorac Cardiovasc Surg. 2004;127:1808-10.

7. Ye C, Chang G, Li S, et al. Endovascular stent-graft treatment for Stanford type A aortic dissection. Eur J Vasc Endovasc Surg. 2011;42:787-94.

8. Vallabhajosyula P, Gottret J-P, Bavaria JE, Desai ND, Szeto WY. Endovascular repair of the ascending aorta in patients at high risk for open repair. J Thorac Cardiovasc Surg. 2015; 149:S144-50.

9. Nienaber CA, Kische S, Rehders TC, et al. Rapid pacing for better placing: comparison of techniques for precise deployment of endografts in the thoracic aorta. J Endovasc Ther. 2007;14:506-12.

10. Bossone E, Evangelista A, Isselbacher E, et al. Prognostic role of transesophageal echocardiography in acute type A aortic dissection. Am Heart J. 2007;153:1013-20.

11. Nienaber CA, Sakalihasan N, Clough RE, et al. Thoracic endovascular aortic repair (TEVAR) in proximal (type A) aortic dissection: Ready for a broader application? J Thorac Cardiovasc Surg. 2017;153:S3-S11.

12. Koschyk DH, Nienaber CA, Knap M, et al. How to guide stentgraft implantation in type B aortic dissection? Comparison of angiography, transesophageal echocardiography, and intravascular ultrasound. Circulation. 2005;112:I260-4.

13. Moon MC, Greenberg RK, Morales JP, et al. Computed tomography-based anatomic characterization of proximal aortic dissection with consideration for endovascular candidacy. J Vasc Surg. 2011;53:942-9.

14. Roselli EE, Idrees J, Greenberg RK, Johnston DR, Lytle BW. Endovascular stent grafting for ascending aorta repair in highrisk patients. J Thorac Cardiovasc Surg. 2015;149:144-51.

15. Khoynezhad A, White RA, Koopmann MC, Kopchok GE, Walot I, Donayre CE. Ascending Aortic Endovascular Repair (AA EVR): Results of FDA-approved PS-IDE feasibility study. J Vasc Surg. 2015;62:529-30.

16. Rampoldi V, Trimarchi S, Eagle KA, et al. Simple risk models to predict surgical mortality in acute type A aortic dissection: the International Registry of Acute Aortic Dissection score. Ann Thorac Surg. 2007;83:55-61.

17. Diethrich EB, Ghazoul M, Wheatley GH, et al. Surgical correction of ascending type A thoracic aortic dissection: simultaneous endoluminal exclusion of the arch and distal aorta. J Endovasc Ther. 2005;12:660-6.

18. Dobrilovic N ,Elefteriades JA. Stenting the descending aorta during repair of type A dissection: technology looking for an application? J Thorac Cardiovasc Surg. 2006;131:777-8.

19. Ueda T, Fleischmann D, Dake MD, Rubin GD, Sze DY. Incomplete endograft apposition to the aortic arch: bird-beak configuration increases risk of endoleak formation after thoracic endovascular aortic repair. Radiology. 2010;255:645-52.

20. Metcalfe MJ, Karthikesalingam A, Black SA, Loftus IM, Morgan $\mathrm{R}$, Thompson MM. The first endovascular repair of an acute type A dissection using an endograft designed for the ascending aorta. J Vasc Surg. 2012;55:220-2.

21. Lu Q, Feng J, Zhou J, et al. Endovascular repair of ascending aortic dissection: a novel treatment option for patients judged unfit for direct surgical repair. J Am Coll Cardiol. 2013;61:1917-24.

22. Ronchey S, Serrao E, Alberti V, et al. Endovascular stenting of the ascending aorta for type A aortic dissections in patients at high risk for open surgery. Eur J Vasc Endovasc Surg. 2013;45:475-80.

23. Nienaber CA, Fattori R, Lund G, et al. Nonsurgical reconstruction of thoracic aortic dissection by stent-graft placement. N Engl J Med. 1999;340:1539-45.
24. Dake MD, Kato N, Mitchell RS, et al. Endovascular stent-graft placement for the treatment of acute aortic dissection. N Engl J Med. 1999;340:1546-52.

25. Wang ZG, Massimo CG, Li M, et al. Deployment of endograft in the ascending aorta to reverse type A aortic dissection. Asian J Surg. 2003;26:117-9.

26. Zhang H, Li M, Jin W, Wang Z. Endoluminal and surgical treatment for the management of Stanford Type A aortic dissection. Eur J Cardiothorac Surg. 2004;26:857-9.

27. Zimpfer D, Czerny M, Kettenbach J, et al. Treatment of acute type A dissection by percutaneous endovascular stent-graft placement. Ann Thorac Surg. 2006;82:747-9.

28. Comas GM, Leshnower BG, Halkos ME, et al. Acute type A dissection: impact of antegrade cerebral perfusion under moderate hypothermia. Ann Thorac Surg. 2013;96:2135-41.

29. Tsai TT, Evangelista A, Nienaber CA, et al. Partial thrombosis of the false lumen in patients with acute type B aortic dissection. $\mathrm{N}$ Engl J Med. 2007;357:349-59.

30. Kim T-H, Song S-W, Lee K-H, Baek M-Y, Yoo K-J. Effects of false lumen procedures on aorta remodeling of chronic DeBakey IIIb aneurysm. Ann Thorac Surg. 2016;102:1941-7.

31. Gysi J, Schaffner T, Mohacsi P, Aeschbacher B, Althaus U, Carrel T. Early and late outcome of operated and non-operated acute dissection of the descending aorta. Eur J Cardiothorac Surg. 1997;11:1163-9.

32. Haverich A, Miller DC, Scott WC, et al. Acute and chronic aortic dissections--determinants of long-term outcome for operative survivors. Circulation. 1985;72:II22-34.

33. Umana JP, Lai DT, Mitchell RS, et al. Is medical therapy still the optimal treatment strategy for patients with acute type B aortic dissections? J Thorac Cardiovasc Surg. 2002;124:896-910.

34. Bernard Y, Zimmermann H, Chocron S, et al. False lumen patency as a predictor of late outcome in aortic dissection. Am J Cardiol. 2001;87:1378-82.

35. Oikonomou K, Katsargyris A, Ritter W, Spinelli D, Seto Y, Verhoeven EL. Endovascular management of chronic post-dissection aneurysms. Ann Cardiothorac Surg. 2014;3:307-13.

36. Roselli EE, Sepulveda E, Pujara AC, Idrees J, Nowicki E. Distal landing zone open fenestration facilitates endovascular elephant trunk completion and false lumen thrombosis. Ann Thorac Surg. 2011;92:2078-84.

37. Kolbel T, Lohrenz C, Kieback A, Diener H, Debus ES, LarenaAvellaneda A. Distal false lumen occlusion in aortic dissection with a homemade extra-large vascular plug: the candy-plug technique. J Endovasc Ther. 2013;20:484-9.

38. Marone EM, Leopardi M, Bertoglio L, Mascia D, Chiesa R. Original off-label endovascular solution to occlude false lumen rupture in chronic type B aortic dissection. Ann Vasc Surg. 2017;40:299 e1-299 e5.

39. Van den Eynde W, Verbist J. A growing type I TAAA after treatment with a multilayer stent. Eur J Vasc Endovasc Surg. 2017;54:438.

40. Ke K, Zheng H, Yang W. Is multilayer bare stent safe or effective for the treatment of aortic aneurysms? A meta-analysis with early and mid-term outcomes. Ann Vasc Surg. 2017;40:112-9.

41. Wojtaszek M, Wnuk E, Maciag R, Lamparski K, Korzeniowski $\mathrm{K}$, Rowinski O. Promoting false-lumen thrombosis after thoracic endovascular aneurysm repair in type B aortic dissection by selectively excluding false-lumen distal entry tears. J Vasc Interv Radiol. 2017;28:168-75.

42. Hofferberth SC, Nixon IK, Mossop PJ. Aortic false lumen thrombosis induction by embolotherapy (AFTER) following endovascular repair of aortic dissection. J Endovasc Ther. 2012;19:538-45.

43. Yuan X, Mitsis A, Mozalbat D, Nienaber CA. Novel endovascular management of proximal type A (DeBakey II) aortic 
dissection with a patent foramen ovale occluder. J Endovasc Ther. 2017;24:809-13.

44. Henrikson O, Roos H, Falkenberg M. Ethylene vinyl alcohol copolymer (Onyx) to seal type 1 endoleak A new technique. Vascular. 2011;19:77-81.

45. Marcelin C, Le Bras Y, Petitpierre F, et al. Safety and efficacy of embolization using $\operatorname{Onyx}((\mathrm{R}))$ of persistent type II endoleaks after abdominal endovascular aneurysm repair. Diagn Interv Imaging. 2017;98:491-7.

46. Clough RE, Nienaber CA. Evidence for and risks of endovascular treatment of asymptomatic acute type B aortic dissection. J Cardiovasc Surg. 2017;58:270-7.

47. Sailer AM, van Kuijk SMJ, Nelemans PJ, et al. Computed tomography imaging features in acute uncomplicated stanford TypeB aortic dissection predict late adverse events. Circ Cardiovasc Imaging. 2017;10:e005709.

48. Kolbel T, Reiter B, Schirmer J, Wipper S, Detter C, Debus ES, Reichenspurner H. Customized transapical thoracic endovascular repair for acute type A dissection. Ann Thorac Surg. 2013;95:694-6.

49. Luo C-M, Wang S-S, Chi N-H, Wu I-H. Transapical endovascular repair with a table-tailored endograft to treat ascending aortic dissection. J Card Surg. 2014;29:824-6.

50. Guo W, Liu X, Liang F, et al. Transcarotid artery endovascular reconstruction of the aortic arch by modified bifurcated stent graft for Stanford type A dissection. Asian J Surg. 2007;30:290-5.

51. Mangialardi N, Ronchey S, Malaj A, et al. Case report of an endovascular repair of a residual type A dissection using a not $\mathrm{CE}$ not FDA-approved Najuta thoracic stent graft system. Medicine (Baltimore). 2015;94:e436.

52. Sabik JF, Lytle BW, McCarthy PM, Cosgrove DM. Axillary artery: an alternative site of arterial cannulation for patients with extensive aortic and peripheral vascular disease. J Thorac Cardiovasc Surg. 1995;109:885-90.

53. Berretta P, Patel HJ, Gleason TG, et al. IRAD experience on surgical type A acute dissection patients: results and predictors of mortality. Ann Cardiothorac Surg. 2016;5:346-51.

54. Hagan PG, Nienaber CA, Isselbacher EM, et al. The International Registry of Acute Aortic Dissection (IRAD): new insights into an old disease. JAMA. 2000;283:897-903.

55. Geisbusch P, Hoffmann S, Kotelis D, Able T, Hyhlik-Durr A, Bockler D. Reinterventions during midterm follow-up after endovascular treatment of thoracic aortic disease. J Vasc Surg. 2011;53:1528-33.

56. Ongstad SB, Miller DF, Panneton JM. The use of EndoAnchors to rescue complicated TEVAR procedures. J Cardiovasc Surg. 2016;57:716-29.

57. Menon RS, Muetterties C, Moser GW, Wheatley GH 3rd. Endoanchor stenting for the repair of a type I endoleak in the aortic arch following the endovascular repair of a Kommerrell's diverticulum. J Card Surg. 2016;31:541-3.

58. Tse LW, Lindsay TF, Roche-Nagle G, Oreopoulos GD, Ouzounian M, Tan KT. Radiofrequency in situ fenestration for aortic arch vessels during thoracic endovascular repair. J Endovasc Ther. 2015;22:116-21.

59. Li Z, Lu Q, Feng R, et al. Outcomes of endovascular repair of ascending aortic dissection in patients unsuitable for direct surgical repair. J Am Coll Cardiol. 2016;68:1944-54.

Publisher's note Springer Nature remains neutral with regard to jurisdictional claims in published maps and institutional affiliations. 